\title{
The Evolution of Marine Larval Dispersal Kernels in Spatially Structured Habitats: Analytical Models, Individual-Based Simulations, and Comparisons with Empirical Estimates
}

\author{
Allison K. Shaw, ${ }^{1, \star}$ Cassidy C. D’Aloia, ${ }^{2, \dagger}$ and Peter M. Buston ${ }^{3}$ \\ 1. Department of Ecology, Evolution, and Behavior, University of Minnesota, Saint Paul, Minnesota 55108; 2. Woods Hole Oceanographic \\ Institution, Biology Department, Woods Hole, Massachusetts 02543; 3. Boston University, Department of Biology and Marine Program, \\ Boston, Massachusetts 02215
}

Submitted April 2, 2018; Accepted November 2, 2018; Electronically published January 17, 2019

Online enhancements: appendixes. Dryad data: http://dx.doi.org/10.5061/dryad.s3j9074.

\begin{abstract}
AвSTRACт: Understanding the causes of larval dispersal is a major goal of marine ecology, yet most research focuses on proximate causes. Here we ask how ultimate, evolutionary causes affect dispersal. Building on Hamilton and May's classic 1977 article "Dispersal in Stable Habitats," we develop analytic and simulation models for the evolution of dispersal kernels in spatially structured habitats. First, we investigate dispersal in a world without edges and find that most offspring disperse as far as possible, opposite the pattern of empirical data. Adding edges to our model world leads to nearly all offspring dispersing short distances, again a mismatch with empirical data. Adding resource heterogeneity improves our results: most offspring disperse short distances with some dispersing longer distances. Finally, we simulate dispersal evolution in a real seascape in Belize and find that the simulated dispersal kernel and an empirical dispersal kernel from that seascape both have the same shape, with a high level of short-distance dispersal and a low level of long-distance dispersal. The novel contributions of this work are to provide a spatially explicit analytic extension of Hamilton and May's 1977 work, to demonstrate that our spatially explicit simulations and analytic models provide equivalent results, and to use simulation approaches to investigate the evolution of dispersal kernel shape in spatially complex habitats. Our model could be modified in various ways to investigate dispersal evolution in other species and seascapes, providing new insights into patterns of marine larval dispersal.
\end{abstract}

Keywords: biological oceanography, dispersal kernel, evolutionarily stable strategy, larval dispersal, marine ecology, population connectivity.

\footnotetext{
* Corresponding author; email: ashaw@umn.edu.

$\dagger$ Present address: University of New Brunswick, Department of Biological Sciences, Saint John, New Brunswick E2L 4L5 Canada.

ORCIDs: Shaw, http://orcid.org/0000-0001-7969-8365; D'Aloia, http://orcid .org/0000-0002-0824-0151.
}

Am. Nat. 2019. Vol. 193, pp. 424-435. (C) 2019 by The University of Chicago. 0003-0147/2019/19303-58374\$15.00. All rights reserved.

DOI: $10.1086 / 701667$
Understanding the patterns, causes, and consequences of larval dispersal is a major goal of marine ecology and biological oceanography (Cowen et al. 2002; Botsford et al. 2009). Patterns of larval dispersal determine the probability of larval exchange between populations, which in turn has major consequences for population dynamics (Botsford et al. 2001; Hastings and Botsford 2006) and genetic differentiation within metapopulations (Taylor and Hellberg 2003; D’Aloia et al. 2015). Consequently, dispersal is an important consideration in fisheries management and reserve design (Sala et al. 2002; Sale et al. 2005).

Larval dispersal was once assumed to be extensive, leading to demographically and genetically open marine populations (Roughgarden et al. 1985; Scheltema 1986; Roberts 1997). However, interdisciplinary efforts have revealed heterogeneity among species in the scale of marine dispersal (Kinlan and Gaines 2003). More recently, complete descriptions of dispersal patterns have emerged, drawing on genetic parentage analysis (D'Aloia et al. 2015; Williamson et al. 2016; Almany et al. 2017) or spatially extensive data on reproduction and settlement (Hameed et al. 2016). Collectively, these studies provide evidence that diverse marine species exhibit leptokurtic patterns of dispersal, with a large number of offspring staying relatively close to home and a smaller number of offspring dispersing long distances. The qualitative similarity in these empirical dispersal patterns, for marine species with varied life-history traits and habitat associations, raises the intriguing question of what generates these patterns.

Generally, when marine ecologists and biological oceanographers consider the causes of variation in patterns of dispersal, they focus on proximate causes such as adult spawning characteristics, larval behavior, larval duration, and oceanographic flow fields (Treml et al. 2015). With 
this approach, biological trait data are used to parameterize coupled biophysical models that track simulated larvae as they disperse from spawning to settlement sites on ocean currents (Paris et al. 2005; Cowen 2006; Paris et al. 2013). These mechanistic models enable predictions of how biological and physical drivers could interact to affect larval dispersal patterns. Although these models have the potential to achieve a high level of biological realism, there have been few direct comparisons between biophysical models and empirical dispersal kernels. In one published case of direct comparison, the biophysical model overestimates dispersal (Hameed et al. 2016), indicating that there is room for complementary approaches.

An alternative approach is to consider the ultimate causes of variation in patterns of larval dispersal, for example, direct selection for adult or larval dispersal strategies (sensu Hamilton and May 1977). There is an extensive literature on the evolution of reproductive strategies (e.g., small eggs and planktotrophy vs. large eggs and lecithotrophy) in marine invertebrates (Vance 1973; Christiansen and Fenchel 1979; McEdward 1997; Levitan 2000), which may have indirect consequences for the pattern of dispersal. Indeed, building on this idea, some have argued for the idea that marine larval dispersal may be a nonadaptive effect (sensu Williams 1966) of natural selection on other traits, that is, a by-product of selection for traits with other functions such as predator avoidance or larval feeding (Strathmann et al. 2002; Pringle et al. 2014; Burgess et al. 2015). Those studies that have considered the evolution of larval dispersal strategies directly have focused on how habitat fragmentation affects the evolution of dispersal distance (Baskett et al. 2007) or larval movement (Dytham and Simpson 2007). Still, there are relatively few theoretical investigations of the idea that marine larval dispersal may be adaptive, that is, a direct product of selection for adult or larval dispersal strategies, and such investigations may provide new insights.

Much of the current theory surrounding the evolution of dispersal builds on the framework developed by Hamilton and May (1977), with terrestrial systems in mind either explicitly or implicitly. Here dispersal probability evolves to minimize kin competition and maximize exploitation of available habitat (see Ronce 2007 for a review of other mechanisms, including inbreeding avoidance and bet hedging in ephemeral habitats). Using an evolutionarily stable strategy approach, Hamilton and May (1977) showed analytically that natural selection will favor parents that allocate a high proportion of their offspring to dispersal, even in saturated environments in which the mortality of dispersing offspring is extremely high (Hamilton and May 1977). Subsequent models exploring the evolution of dispersal have typically shifted to an individual-based simulation approach to explore the factors that influence dispersal rate (Travis and Dytham 1998, 1999), dispersal distance (Murrell et al. 2002; Shaw et al.

2014), or, more rarely, dispersal kernel shape (Hovestadt et al. 2001).

Here we consider the evolution of marine larval dispersal kernels in spatially structured habitats. Our goal is to provide new insights into the causes of variation in marine larval dispersal and to investigate whether selection for dispersal can explain observed patterns of dispersal. We begin with a simple model to illustrate the evolution of dispersal strategies in a spatially structured, homogeneous world without edges (fig. 1A) and compare and contrast our results with those of Hamilton and May (1977). Using this model as a foundation, we proceed to model the evolution of dispersal strategies in a homogeneous world with edges (fig. $1 B$ ) and a heterogeneous world with edges (fig. $1 C$ ). Finally, we model the evolution of dispersal strategies in a real spatially structured habitat (the Belize Barrier Reef; fig. 1D) and compare the predictions of our model to the empirically derived dispersal kernel of the neon goby Elacatinus lori, a coral reef fish endemic to the region (D'Aloia et al. 2015).

A
B
C

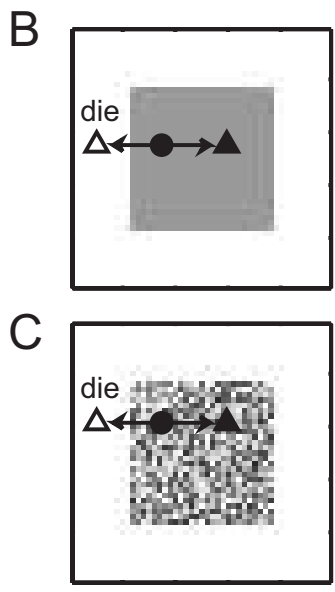

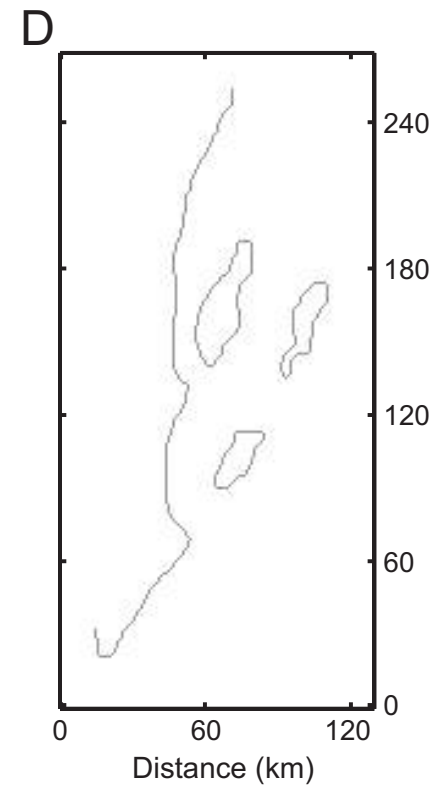

240

120

60

Figure 1: Environmental structures: $A$, Homogeneous world without edges (wrapping boundaries; any individual dispersing off one edge is mapped back via the opposite edge). $B$, Homogeneous world with edges (nonwrapping boundaries; individuals dispersing off one edge die). $C$, Heterogeneous world with edges (nonwrapping boundaries; heterogeneous breeding resources). $D$, Real seascape. Viable sites are gray (shaded proportional to breeding resources), nonviable sites are white, and the edge of the world is denoted with a thick black line. Two examples of possible dispersal outcomes are each shown in $A-C$, where circles are starting locations, open triangles are unsuccessful postdispersal locations, and filled triangles are successful settlement locations. 


\section{Homogeneous Worlds without Edges}

We first consider an analytic model of a homogeneous world without edges, similar to that developed by Hamilton and May (1977; see also app. A; apps. A-E are available online). We assume that the world consists of $S$ identical sites, arranged in a two-dimensional lattice with wrapping boundaries (fig. 1A). Each site supports one adult, and adults die immediately after reproduction. Newborn individuals either disperse or stay at the natal site. Dispersing individuals survive with probability $p$, while all nondispersing individuals survive (i.e., until they compete for a site).

We start by considering a general case. A fraction $v$ of newborn offspring disperses and can reach one of $z$ sites (where $z \leq S$, the total number of sites). The remaining $1-v$ offspring stay at the natal site. We look for the evolutionarily stable strategy (ESS) dispersal probability $v^{*}$. The fitness of a mutant playing dispersal strategy $v^{\prime}$ in a population of individuals each playing strategy $\bar{v}$ will be

$$
\begin{aligned}
w\left(v^{\prime}, \bar{v}\right)= & \frac{1-v^{\prime}}{\left(1-v^{\prime}\right)+(1 / z)[z \bar{v} p]} \\
& +z \frac{(1 / z)\left[v^{\prime} p\right]}{(1-\bar{v})+(1 / z)\left[v^{\prime} p+(z-1) \bar{v} p\right]} .
\end{aligned}
$$

The mutant's fitness is just the sum of two components: (1) the probability that the mutant retains its home site, that is, that one of the natal mutant offspring $1-v^{\prime}$ wins rather than one of the $\bar{v}$ offspring dispersing in from other sites $(\bar{v} p$, the number of residents who successfully disperse from $z$ other sites, a fraction $1 / z$ of which arrives at the mutant's site); and (2) the probability that the mutant wins one of the other $z$ sites, that is, that one of the mutant offspring at each of the $z$ sites $\left(v^{\prime} p\right) / z$ wins rather than the natal offspring $1-\bar{v}$ or one of the other $(z-1) \bar{v} p / z$ offspring dispersing in from other sites. To find the ESS, we look for critical points by differentiating equation (1) with respect to $v^{\prime}$, evaluating it at $v^{\prime}=\bar{v}=v^{*}$, setting it to zero and solving for $v^{*}$. This gives us the ESS dispersal probability

$$
v^{*}=\frac{z}{z(2-p)+p} \text {. }
$$

This ESS, as with all those presented below, can be thought of either as a parental decision rule, with parents allocating a proportion $v^{*}$ of their offspring to dispersal or, as an offspring decision rule, with offspring dispersing with probability $v^{*}$ (Maynard Smith 1982; Gross 1996). When dispersal is global and the number of reachable sites is large $(z, S \rightarrow \infty)$, we recover Hamilton and May's (1977) result of $v^{*}=$ $1 /(2-p)$ (fig. 2, dashed lines), and selection will favor parents that allocate nearly all of their offspring to dispersal when $p$ is large.

When dispersal is local, dispersing individuals reach fewer sites than with global dispersal and the fraction of offspring that disperses declines (fig. $2 A$ ). If dispersing individuals travel a distance of 1 site (and thus $z=4$ ), the ESS dispersal probability is $v^{*}=4 /(8-3 p)$ (fig. $2 A$, line; app. B). Intuitively, since all sites are equal, when all dispersers survive $(p=1)$, the four sites reachable via dispersal and the home site all have the same expected payoff. In this case, selection will favor parents that allocate onefifth of their offspring to the nondispersing tactic and four-fifths of their offspring to the dispersing tactic. If dispersing individuals can travel distances of 1 or 2 sites, twice as many offspring should disperse a distance of 2 as disperse a distance of 1 , because those traveling a distance of 2 can reach twice as many sites (fig. $2 B$, lines; app. C). By extension, we can consider dispersing individuals traveling distances $1,2, \ldots, d_{\max }$ (app. D). Since those offspring dispersing longer distances can reach more potential sites, parents should always allocate the largest proportion of offspring to the farthest dispersal distance.

In the next section, we move to individual-based model (IBM) simulations to evolve dispersal strategies in more complex environments (see also app. E). To confirm that our IBM results are in fact the ESS, we first simulated our IBM on a lattice of $S=1,024(32 \times 32)$ viable sites. These simulations produced evolved dispersal strategies that closely match the analytic ESS (fig. 2, bars vs. lines).

\section{IBM Methods}

The core of our article is a set of IBMs, described below following the revised overview, design concepts, and details (ODD) protocol for IBMs (Grimm et al. 2006, 2010) and deposited in the Dryad Digital Repository: http://dx.doi .org/10.5061/dryad.s3j9074 (Shaw et al. 2018).

\section{Overview}

Purpose. The purpose of the model is to determine what dispersal strategies evolve in different spatially structured environments.

Entities, State Variables, and Scales. The model consists of an environment and agents. See table 1 for all model parameters. The environment is a two-dimensional lattice of hospitable/inhospitable sites. The model agents are asexual individuals, each characterized by a dispersal strategy and the identity of the site it inhabits. An individual's dispersal strategy, $K$, is its dispersal kernel, defined here as the set of dispersal distance tactics $d=\left\{0,1,2, \ldots, d_{\max }\right\}$, each played with probability $k_{d}$. The set of $k_{d}$ sums to 1 .

Process Overview and Scheduling. Time is modeled as discrete, nonoverlapping generations, per Hamilton and May 


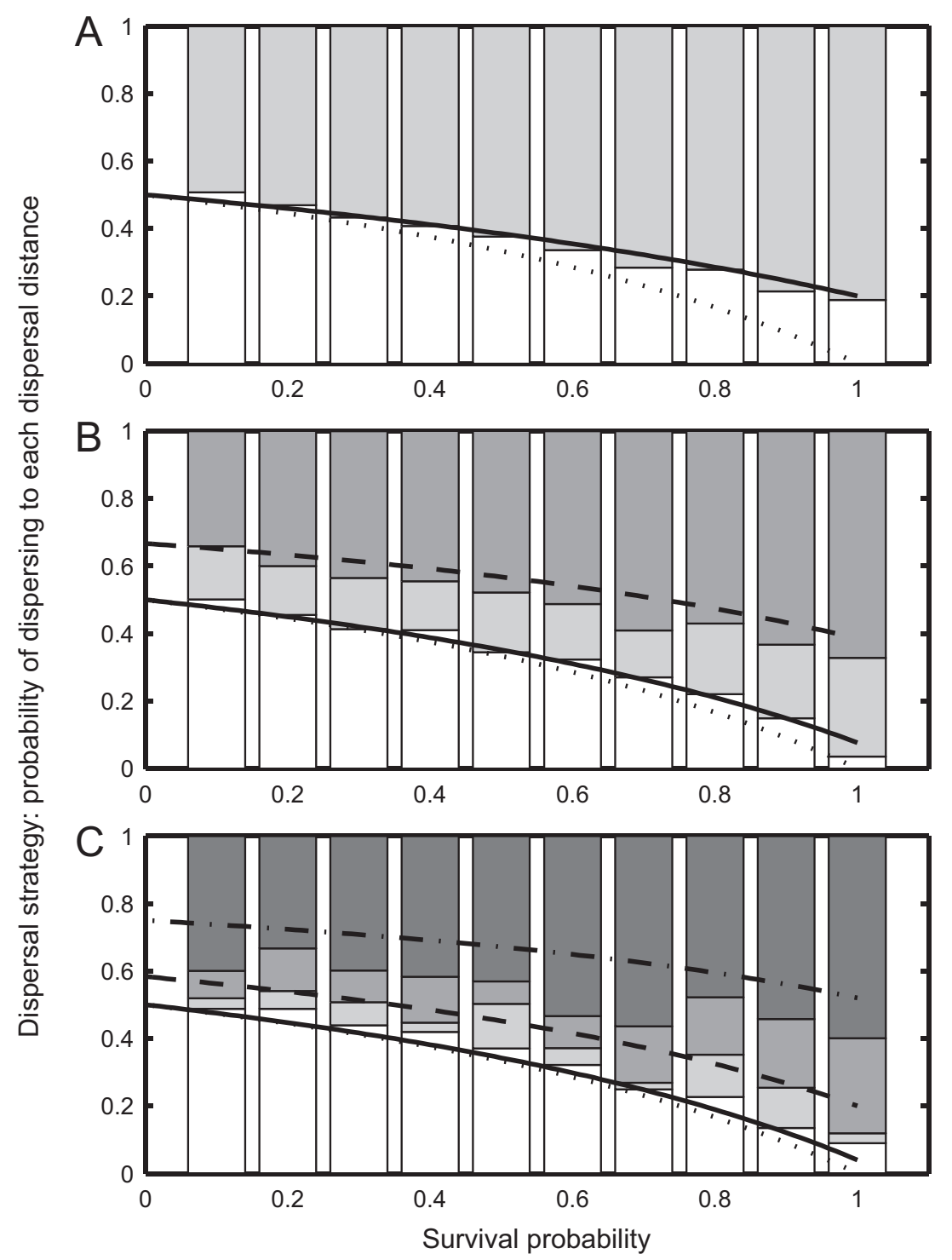

\begin{tabular}{l|l|l|l|l|}
\hline Dispersal distance: & 0 & 1 & 2 & 3 \\
\hline
\end{tabular}

Figure 2: Homogeneous worlds without edges. Shown is the evolutionarily stable dispersal strategy, both analytic (lines) and individualbased (bars) models, as a function of the probability of surviving the act of dispersal $(p)$, with $b=10$ as the reproductive payoff for each viable site: $d=\{0,1\}(A), d=\{0,1,2\}(B)$, and $d=\{0,1,2,3\}(C)$. Lines indicate the evolutionarily stable strategy for distance 1 (solid, $A-C$ ), 2 (dashed, $B-C$ ), 3 (dashed-dotted, $C$ ), and, for comparison, the ESS when dispersal is global (dotted, $A-C$; eq. [1]).

(1977). Within each generation, the processes of reproduction, mutation, dispersal, survival, settlement, and competition occur sequentially. At the start of a generation, every individual produces $b$ offspring and then dies. Each offspring is born into their parent's site and inherits their parent's dispersal strategy (see "Mutation"). Each offspring disperses by traveling a distance according to their dispersal tactic and lands at a site that distance away (see "Stochas- ticity"). Offspring that disperse out of the natal site have a fixed probability of surviving, $p$. Given that mortality can either increase (e.g., increased predation with time spent dispersing) or decrease (e.g., increased escape from natural enemies; Janzen 1970; Connell 1971) with distance traveled during dispersal (Bonte et al. 2012), we make the simplifying assumption that survival is constant with distance traveled. Offspring that survive dispersal either land in one of the vi- 
Table 1: Model parameters and values

\begin{tabular}{lll}
\hline Parameter & \multicolumn{1}{c}{ Meaning } & \multicolumn{1}{c}{ Simulation value } \\
\hline$b_{i}$ & No. offspring produced by an individual in patch $i$ & Varied: 10, $\mathrm{U}(7,13), \mathrm{U}(3,17)$, or $\mathrm{U}(0,20)$ \\
$d_{\max }$ & Maximum dispersal distance & $1,2,3$, or 30 \\
$d$ & Dispersal tactic (distance) & $\left\{0,1, \ldots, d_{\text {max }}\right\}$ \\
$k_{d}$ & Probability of dispersing a distance $d$; the sum of $k_{d}$ over all $d$ is 1 & Evolved \\
$K$ & Dispersal strategy, i.e., set of all $k_{d}$ & $k_{d}$ \\
$p$ & Probability that an individual survives the act of dispersing & $\{.1, .2, \ldots, .9,1\}$ \\
$S$ & Total no. viable sites in the world & 1,024 (unbounded, bounded) or 507 (seascape) \\
$\delta$ & Amount to move from one dispersal probability to another & .001 \\
& during mutation & \\
\hline
\end{tabular}

able sites in the environment and survive or, failing to do so, die. After settlement, any viable site that contains more than one individual undergoes competition, with only one individual surviving in that site.

\section{Design Concepts}

Basic Principles. In this model, dispersal strategies evolve over generations in response to the environmental structure (i.e., the spatial configuration of habitable sites and site quality).

Emergence. The key model output is the dispersal strategy that evolves over time within the population, that is, a probability density function representing the proportion of offspring allocated to each dispersal distance.

Mutation. Each offspring inherits its parent's dispersal strategy (a vector of probabilities of the same length as $d$ summing to 1) with some mutation, using a method similar to that of Hovestadt et al. (2001). Mutation removes a small amount $(\delta=0.001)$ from a randomly chosen vector element (bounded at zero to avoid negative probabilities), and this amount is then added to a distinct randomly chosen vector element. In sum, with this mutation algorithm, an individual's final dispersal strategy, $K^{\prime}$, is given by

$$
\begin{aligned}
& k_{i}^{\prime}=k_{i}-\min \left(\delta, k_{i}\right), \\
& k_{j}^{\prime}=k_{j}+\min \left(\delta, k_{i}\right), \\
& k_{l}^{\prime}=k_{l} \quad \text { for } \quad l \neq i \text { or } j .
\end{aligned}
$$

Interaction. Individuals only interact through competition, which occurs when more than one individual settles in the same viable site in the environment.

Stochasticity. The model processes of mutation, dispersal, survival, and competition each include stochasticity. We use the inverse cumulative distribution method to generate random survival during dispersal (with probability $p$ ) and random dispersal distances. We use the Von Neumann neighborhood to describe the set of possible sites where a dispersing individual can land (fig. C1; figs. A1, B1, C1, E1-E3 are available online). An individual dispersing a distance 1 will land in one of the four sites directly adjacent to its starting site, an individual dispersing a distance 2 will land in one of eight sites (four in the cardinal directions and four along the diagonals), and generally, an individual dispersing a distance $i$ will land in one of $4 i$ possible sites. One of these sites is chosen at random for each dispersing individual. Of the individuals who colonize the same site, a single winner of the competition is chosen at random and all other individuals die.

Observation. At the start of every generation (after competition and before reproduction), the average and standard deviation (taken across the population) value of each of the $k_{d}$ probabilities is stored. All individuals start by always staying at the natal site (dispersing a distance of 0 ). Simulations were then run for a sufficiently long time, until the average value of each $k_{d}$ was stable (figs. E1, E2): $10^{5}$ generations for most simulations and $2 \times 10^{5}$ for seascape simulations. The average dispersal strategy in the population at this point, $K^{*}$, was taken to be the evolutionarily stable strategy. The final $k_{d}$ of all individuals was stored and showed that it was never the case that several distinct strategies evolved and coexisted in the population.

\section{Homogeneous Worlds with Edges}

While the analytic models for homogeneous worlds without edges help build our intuition regarding the proportion of offspring that parents should allocate to each distance (or the probability that offspring disperse to each distance), their predictions create a paradox. Specifically, the predictions run counter to most empirically measured 
dispersal kernels (Jordano et al. 2007; D’Aloia et al. 2015), where more offspring are typically allocated to shorter dispersal distances. What is driving this discrepancy between model predictions and empirical data? In the real world, in contrast to the preceding models, habitat is finite and boundaries are rarely wrapping, creating the distinct possibility of dispersing too far and landing in unsuitable habitat (either locally, outside a suitable site, or globally, outside of the biogeographic range). To determine how this risk of dispersing too far shapes the evolution of dispersal strategies, we constructed a set of models where the environment has edges. That is, in these models, it is possible for individuals to disperse past the edge of viable habitat and land in a nonviable site where they die. Edges are a realistic feature of a diverse set of marine habitats and can be present when there are hard transitions between habitat types (e.g., mangrove to seagrass bed) or when a singular habitat type is patchy and surrounded by unsuitable habitat (e.g., hydrothermal vents or coral reefs).

Here we use individual-based simulations to evolve dispersal strategies. This model is structurally the same as the above one except that the $S$ viable sites are surrounded on all sides by nonviable sites. In this model, dispersal strategies are shaped by two sources of dispersal mortality (failure to survive dispersal and failure to land in a viable site) in contrast with the single mortality source in the model without edges (failure to survive dispersal). Here as above, parents dispersed a larger fraction of their offspring as the probability of surviving dispersal $(p)$ increased (fig. 3). However, given the additional risk of landing in a nonviable site, individuals in a world with edges allocated more offspring to dispersal distance 0 and fewer to distance 3 than individuals in a world without edges (fig. $3 A$ vs. fig. $2 C$ ).

To explore how the risk of landing in a nonviable site shapes the dispersal strategy, we ran simulations with different configurations of viable sites, holding the total number of viable sites constant. Individuals in an $8 \times 128$ site world allocated many fewer offspring to distance 3 than individuals in a $32 \times 32$ site world, even though the total fraction of offspring dispersed was similar (fig. $3 B$ vs. fig. $3 A$ ). Individuals in a $2 \times 512$ site world allocated essentially no offspring to distances 2 or 3 and overall dispersed fewer offspring than individuals in an $8 \times 128$ site world (fig. $3 C$ vs. fig. $3 B$ ). In other words, long-distance dispersal was increasingly disfavored as the habitat configuration became more linear. The risk of landing in a nonviable site had the biggest impact on evolved dispersal strategies when the probability of surviving dispersal was high (large $p$ ). The inclusion of edges (nonviable sites) in a homogeneous world helps to resolve the above paradox and helps to explain why individuals allocate more to shorter distances than longer distances.

\section{Heterogeneous Worlds with Edges}

While the model above helps build our intuition regarding the proportion of effort that parents will allocate to each distance in worlds with edges, resolving the first paradox, it creates a second paradox. Specifically, the predictions still run counter to most empirically derived dispersal kernels, where at least some offspring are allocated to long distances (Jordano et al. 2007; D'Aloia et al. 2015). We have so far assumed that individuals in all sites have the same reproductive payoff. However, breeding resources are often heterogeneous, and thus individuals breeding in different locations may produce different numbers of offspring. To explore the role of resource heterogeneity in shaping dispersal strategies, we constructed a set of models with an environment with edges and spatially heterogeneous resources. In these simulations, the world consisted of $2 \times 512$ viable sites surrounded by nonviable sites. Each viable site was assigned a fixed breeding resource value (number of offspring supported) $b_{i}$ drawn from a uniform random distribution with low, medium, or high levels of spatial heterogeneity. We found that as spatial heterogeneity increased, individuals shifted toward a more uniform dispersal kernel (fig. 4). This shift was especially pronounced for higher survival probabilities.

\section{Real Seascape and Empirical Comparisons}

Finally, we simulated dispersal in the Belizean seascape environment, a long narrow world analogous to the $2 \times 512$ site world described above. This enabled us to compare the model with an empirical study in the same seascape, which used genetic parentage analysis to directly identify dispersal events up to $30 \mathrm{~km}$ from the source (D'Aloia et al. 2015). In this environment, individuals can disperse up to 30 sites away (dispersal tactics $d=0,1,2, \ldots, 30$ ). However, individuals primarily evolved to disperse only 0,1 , or 2 sites away (fig. E3). In contrast to the preceding simulations, we found that the evolved pattern of dispersal over the full $30 \mathrm{~km}$ was similar at multiple levels of spatial resource heterogeneity; we believe this was driven by the fact that resource heterogeneity affected only the evolution of short dispersal distances, so the full dispersal pattern up to $30 \mathrm{~km}$ is rather similar across all heterogeneity simulations. Focusing on the high-heterogeneity simulation as an example, we calculated the net distance traveled by all offspring. To compare the seascape model predictions to empirical data, we considered simulated dispersal events after survival but before within-site competition, as this model stage most closely reflects the life stage at which individuals were sampled in the field (D'Aloia et al. 2015).

We found that both empirical and simulated data show the same kernel shape, with a prevalence of short-distance 


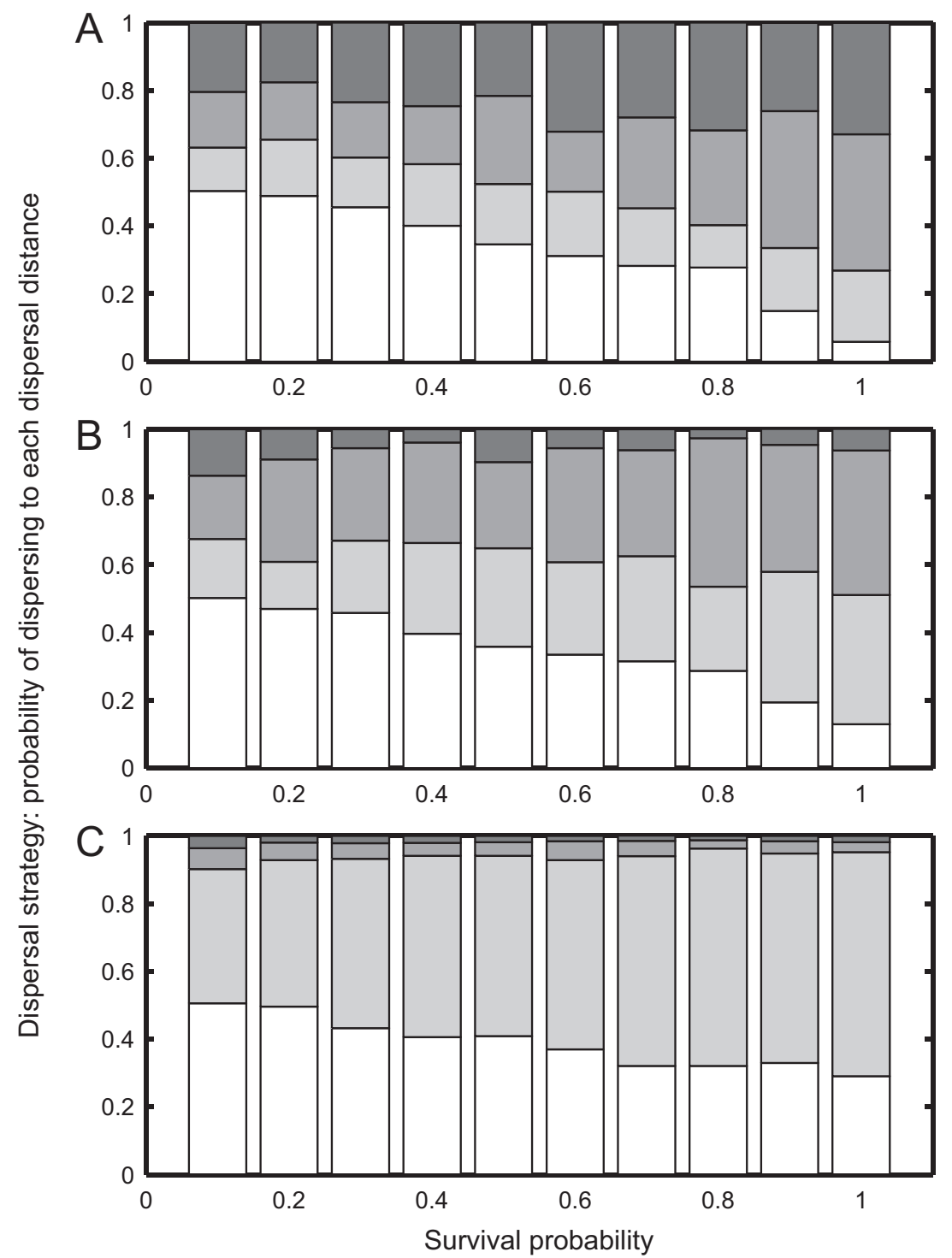

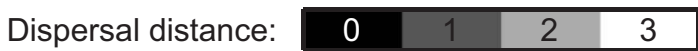

Figure 3: Homogenous worlds with edges. Shown is the individual-based model evolutionarily stable dispersal strategy as a function of dispersal survival $(p)$ for $d=\{0,1,2,3\}$, in a habitat of $32 \times 32(A), 8 \times 128(B)$, and $2 \times 512(C)$ viable sites surrounded by nonviable sites.

dispersal and a small amount of long-distance dispersal (fig. 5A). The modal dispersal distance was less than $1 \mathrm{~km}$ in both data sets: $50 \%$ of simulated larvae stayed at the natal site (i.e., dispersed $<1 \mathrm{~km}$ ), and $50 \%$ of empirical larvae dispersed less than $1.74 \mathrm{~km}$. The simulated data were more strongly right skewed: $99 \%$ of simulated larvae dispersed within $10 \mathrm{~km}$, and 99\% of empirical larvae dispersed within $12.74 \mathrm{~km}$ (and this difference would be amplified if each site supports more than one adult in the model; fig. $5 B$ ). Overall, the simulated and empirical dispersal data both showed a rapid decline in dispersal probabilities with respect to distance from source.

\section{Discussion}

Here we address a critical knowledge gap in the field of marine ecology - investigating what form of larval dispersal kernel will be favored by natural selection. First, building on Hamilton and May (1977), we show that in a world with- 


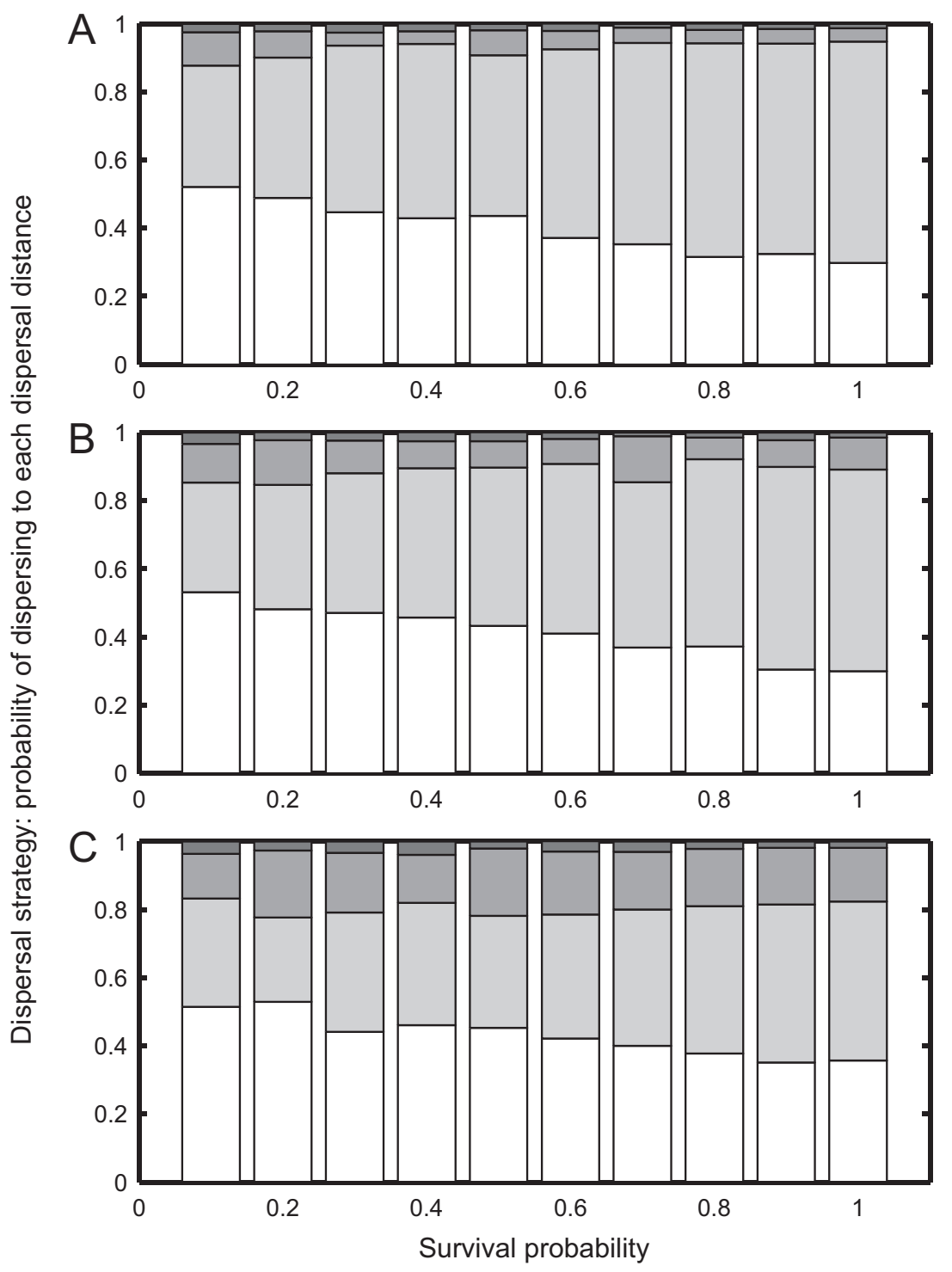

\begin{tabular}{l|l|l|l|l|}
\hline Dispersal distance: & 0 & 1 & 2 & 3 \\
\hline
\end{tabular}

Figure 4: Heterogeneous worlds with edges. Shown is the individual-based model evolutionarily stable dispersal strategy as a function of dispersal survival $(p)$ for $d=\{0,1,2,3\}$ in a habitat of $2 \times 512$ viable sites surrounded by nonviable sites. The reproductive payoff of each viable site was drawn from a distribution with low $(A)$, medium $(B)$, and high $(C)$ variance.

out edges, selection will favor parents that allocate the majority of their offspring to the longest dispersal distance class to minimize sibling competition and maximize exploitation of available habitat. Second, we show that in a narrow world with edges, selection will favor parents that allocate the majority of their offspring to the shortest dispersal distance class. Third, we show that when resource heterogeneity is incorporated into the world with edges, selection will favor parents that allocate offspring more evenly across distance classes, without changing the overall fraction of offspring dispersed. Finally, we show that the dispersal kernel that will evolve in a real, narrow, heterogeneous seascape with edges is of the same general shape as an empirical dispersal kernel measured in that seascape, with modal, median, and long-distance dispersal on the same order of magnitude (D'Aloia et al. 2015). The take-home message is that leptokurtic dispersal kernels can be evolutionarily stable across diverse spatially structured seascapes, 


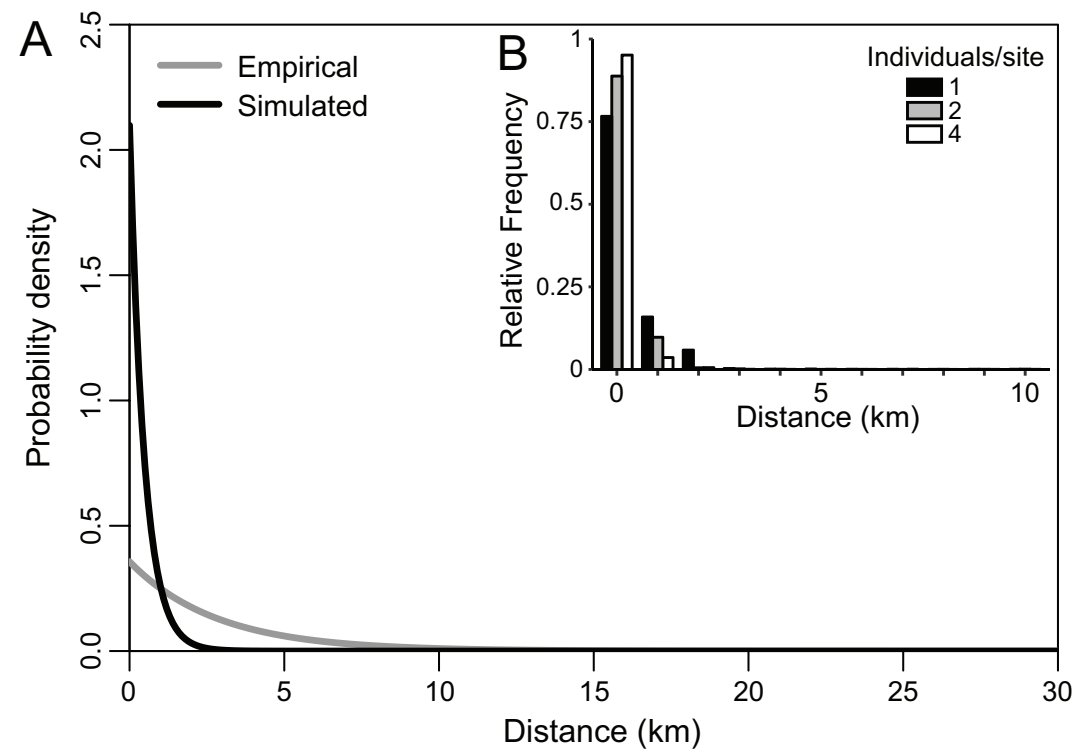

Figure 5: Simulated and empirical dispersal kernels. The simulated kernel represents the evolutionarily stable dispersal strategy from individualbased simulations on the Belizean seascape, with potential dispersal distances up to $d_{\max }=30$, a probability of surviving dispersal $p=1$, and a high level of resource heterogeneity. $A$, Simulated dispersal data (black line) are shown postsurvival. These postsurvival data represent the larvae that dispersed to viable reef sites. Empirical data (gray line) are from the same seascape (D'Aloia et al. 2015). B, inset, relative frequency plots for simulations where each site can support one, two, or four adults.

and this dispersal kernel shape (but not necessarily the scale) is consistent with most published larval dispersal kernels for coral reef fishes (D’Aloia et al. 2015; Williamson et al. 2016; Almany et al. 2017).

This work builds on general evolutionary models of dispersal by focusing on the evolution of dispersal kernel shape, using both analytical and simulation models. In contrast, previous studies typically focus on the evolution of dispersal probability, rate, or distance, using either analytical or simulation methods (Bolker 2010). Our approach, combining analytical and simulation models, enables us to both confirm that our IBM results are, in fact, the ESS and to more closely link our work to previous work using either methodology. Additionally, our approach of modeling the evolution of dispersal kernel shape conveys three distinct advantages: (i) it allows us to compare our findings to existing theory on dispersal rate and distance by extracting these metrics from our richer metric of dispersal kernel shape; (ii) it allows us to compare our findings to existing theory on dispersal kernel shape; and (iii) it also allows us to compare our findings to empirical estimates of dispersal kernels, based on parentage analyses that are now emerging.

Comparing our findings to previous theoretical findings on the evolution of dispersal rate, our results support the idea that increasing spatial variation alone is not sufficient to increase dispersal probability (Hastings 1983; fig. 4).
Turning to consider the evolution of dispersal distance, we confirm the result that more bounded landscapes favor reduced average dispersal distance, both when the shape of the dispersal kernel is specified (Shaw et al. 2014) or left to evolve freely (fig. 3). Turning to consider the evolution of dispersal kernel shape, past simulation studies have shown that random landscapes select for more uniform dispersal kernels (Hovestadt et al. 2001) and that autocorrelated landscapes have differing effects on short- and long-distance dispersal (Bonte et al. 2010). Here we show that narrower landscapes select against longer dispersal distances, an effect that is ameliorated somewhat if there is resource heterogeneity within the suitable habitat. When mortality risk increases with dispersal distance in a homogeneous environment, intermediate dispersal distances are favored (Rousset and Gandon 2002). A detailed exploration of mortality dependent on distance (or time) is beyond the scope of this article, but we expect mortality increasing with distance will select against long-distance dispersal and lead to thinner-tailed dispersal kernels, while mortality decreasing with distance will select for long-distance dispersal and lead to fatter-tailed dispersal kernels. Taken together, our analytic and simulation approach links previous bodies of theory, confirms previous findings, and generates new insights.

Our work complements the work of others who have modeled the evolution of dispersal in the marine environ- 
ment, either explicitly or implicitly. Consistent with our findings, increased habitat fragmentation has been shown to favor the evolution of reduced dispersal distance (Baskett et al. 2007), and increased habitat contiguity has been shown to favor the evolution of increased larval movement (Dytham 2003; Dytham and Simpson 2007). Our model builds on these studies by exploring how dispersal kernel shape evolves in various spatially structured (continuous) habitats. To our knowledge, no previous models have explored the evolution of marine dispersal kernel shapes. In this way, our study parallels advances in empirical data collection in the marine environment, which has shifted away from simple metrics of dispersal, such as mean dispersal distance (Kinlan and Gaines 2003) or self-recruitment (Planes et al. 2009; Harrison et al. 2012; D'Aloia et al. 2013), toward more complete descriptions of dispersal kernels (D’Aloia et al. 2015; Williamson et al. 2016; Almany et al. 2017).

There is one major difference between the simulated and empirical dispersal kernels. The simulated kernel predicts a higher probability of dispersal to short-distance classes and a lower probability of dispersal to long-distance classes than do the empirical kernels, a difference exacerbated with increased site carrying capacity (fig. 5). This pattern is consistent with the hypothesis of Strathmann et al. (2002) and Burgess et al. (2015) that much of observed dispersal is not for dispersal per se but rather is a by-product of selection for offshore movement in the larval stage. However, while we consider this hypothesis stimulating, it does not make quantitative predictions about expected patterns of dispersal, meaning we cannot falsify this nonadaptive hypothesis for the observed pattern of dispersal. More importantly, we consider that there are reasonable modifications of the model that might improve the fit between the simulated and empirical dispersal kernels.

One plausible explanation for this key difference is that our model assumes hard edges where any individual dispersing past the edge dies. It may be the case that larvae can sense they have passed an edge and navigate from nonviable sites to viable (reef) sites, equivalent to cue use during dispersal (Bowler and Benton 2005). We anticipate that including such larval behavior would select for a more uniform dispersal kernel. The logic underlying this idea is that as larval swimming and orientation abilities increase, larvae may be able to be cast further from suitable habitat without that leading to certain death, if they can navigate back to suitable habitat. If this were the case, then interspecific variation in swimming abilities (Fisher et al. 2005; Hogan et al. 2007; Nanninga and Manica 2018) might partially explain interspecific variation in dispersal kernels (D'Aloia et al. 2015; Williamson et al. 2016; Almany et al. 2017).

Confronting these simple evolutionary models with empirical data raises the question of whether parents can control the distance their offspring travel and/or whether larvae can inherit dispersal strategies. Parents may plausibly have some control by varying investment within or across clutches (Kolm 2001) or by timing gamete or larval release with conducive oceanographic conditions (Robertson et al. 1990; Robertson 1991). The extent to which larvae inherit dispersal strategies remains largely unexplored, but there is some evidence for heritable marine dispersal polymorphisms in invertebrates (Toonen and Pawlik 2001) and heritable larval body size in vertebrates (Johnson et al. 2010). More research is needed to disentangle genetic and environmental effects on different species' realized dispersal kernels. Independent of the exact mechanisms, our approach shows that the ESS dispersal strategy from a relatively simple evolutionary model provides a good foundation that could be modified in various ways to predict empirical larval dispersal distances for Elacatinus lori, and this should stimulate further research into the mechanisms.

The framework we develop here could be used to model the evolution of dispersal kernels of different species in different seascapes (or, indeed, landscapes), enabling us to gain new insights into interspecific variation in dispersal kernels. Given a habitat map and a biogeographic range for any species, one could increase the maximum dispersal distance allowed in the simulations until the probability of dispersal to the farthest bins tends to zero. Recent work has shown that codistributed populations of different species can exhibit different dispersal kernels within the same seascape (Almany et al. 2017), suggesting that accounting for range size, habitat requirements, and/or larval behavior may be important extensions of this approach to explain interspecific variation in dispersal kernels. Our framework could also be extended to include other mechanisms shaping the evolution of dispersal (see Ronce 2007) that may be important. Our hope is that this study will stimulate new avenues of research that combine mathematical models and empirical data to explore ultimate causes of larval dispersal and help us make better predictions of population connectivity.

\section{Acknowledgments}

We thank S. Levin, M. Neubert, S. Proulx, L. Sullivan, R. Warner, and several anonymous reviewers for helpful comments. This work was carried out in part using computing resources at the University of Minnesota Supercomputing Institute. The project was supported by a start-up award from the University of Minnesota to A.K.S. and a National Science Foundation award (OCE-1260424) to P.M.B. and colleagues; C.C.D. was supported by the Weston Howland Junior Postdoctoral Scholarship from the Woods Hole Oceanographic Institution. 


\section{Literature Cited}

Almany, G. R., S. Planes, S. R. Thorrold, M. L. Berumen, M. Bode, P. Saenz-Agudelo, M. C. Bonin, et al. 2017. Larval fish dispersal in a coral-reef seascape. Nature Ecology and Evolution 1:1-7.

Baskett, M. L., J. S. Weitz, and S. A. Levin. 2007. The evolution of dispersal in reserve networks. American Naturalist 170:59-78.

Bolker, B. 2010. Evolution of dispersal scale and shape in heterogeneous environments: a correlation equation approach. Pages 231249 in S. Cantrell, C. Cosner, S. Ruan, L. J. Gross, and S. Lenhart, eds. Spatial ecology. Chapman \& Hall, New York.

Bonte, D., T. Hovestadt, and H. J. Poethke. 2010. Evolution of dispersal polymorphism and local adaptation of dispersal distance in spatially structured landscapes. Oikos 119:560-566.

Bonte, D., H. Van Dyck, J. M. Bullock, A. Coulon, M. Delgado, M. Gibbs, V. Lehouck, et al. 2012. Costs of dispersal. Biological Reviews of the Cambridge Philosophical Society 87:290-312.

Botsford, L. W., A. Hastings, and S. D. Gaines. 2001. Dependence of sustainability on the configuration of marine reserves and larval dispersal distance. Ecology Letters 4:144-150.

Botsford, L. W., J. W. White, M.-A. Coffroth, C. B. Paris, S. Planes, T. L. Shearer, S. R. Thorrold, and G. P. Jones. 2009. Connectivity and resilience of coral reef metapopulations in marine protected areas: matching empirical efforts to predictive needs. Coral Reefs 28:327-337.

Bowler, D. E., and T. G. Benton. 2005. Causes and consequences of animal dispersal strategies: relating individual behaviour to spatial dynamics. Biological Reviews of the Cambridge Philosophical Societv 80:205-225.

Burgess, S. C., M. L. Baskett, R. K. Grosberg, S. G. Morgan, and R. R. Strathmann. 2015. When is dispersal for dispersal? unifying marine and terrestrial perspectives. Biological Reviews 91:867-882.

Christiansen, F. B., and T. M. Fenchel. 1979. Evolution of marine invertebrate reproductive patterns. Theoretical Population Biology $16: 267-282$.

Connell, J. H. 1971. On the role of natural enemies in preventing competitive exclusion in some marine animals and in forest trees. Pages 298-312 in P. J. Den Boer and G. R. Gradwell, eds. Dynamics of populations. Centre for Agricultural Publishing and Documentation, Wageningen, Netherlands.

Cowen, R. K. 2006. Scaling of connectivity in marine populations. Science 311:522-527.

Cowen, R. K., G. Gawarkiewicz, J. Pineda, S. Thorrold, and F. Werner. 2002. Population connectivity in marine systems. Report of a Workshop to Develop Science Recommendations for the National Science Foundation, Durango, CO, November 4-6.

D’Aloia, C. C., S. M. Bogdanowicz, R. K. Francis, J. E. Majoris, R. G. Harrison, and P. M. Buston. 2015. Patterns, causes, and consequences of marine larval dispersal. Proceedings of the National Academy of Sciences of the USA 112:13940-13945.

D'Aloia, C. C., S. M. Bogdanowicz, J. E. Majoris, R. G. Harrison, and P. M. Buston. 2013. Self-recruitment in a Caribbean reef fish: a method for approximating dispersal kernels accounting for seascape. Molecular Ecology 22:2563-2572.

D'Aloia, C. C., J. E. Majoris, and P. M. Buston. 2011. Predictors of the distribution and abundance of a tube sponge and its resident goby. Coral Reefs 30:777-786.

Dytham, C. 2003. How landscapes affect the evolution of dispersal behaviour in reef fishes: results from an individual-based model. Journal of Fish Biology 63:213-225.
Dytham, C., and S. Simpson. 2007. Elevated mortality of fish larvae on coral reefs drives the evolution of larval movement patterns. Marine Ecology Progress Series 346:255-264.

Fisher, R., J. M. Leis, D. L. Clark, and S. K. Wilson. 2005. Critical swimming speeds of late-stage coral reef fish larvae: variation within species, among species and between locations. Marine Biology 147:1201-1212.

Grimm, V., U. Berger, F. Bastiansen, S. Eliassen, V. Ginot, J. Giske, J. GossCustard, et al. 2006. A standard protocol for describing individualbased and agent-based models. Ecological Modelling 198:115-126.

Grimm, V., U. Berger, D. L. DeAngelis, J. G. Polhill, J. Giske, and S. F. Railsback. 2010. The ODD protocol: a review and first update. Ecological Modelling 221:2760-2768.

Gross, M. R. 1996. Alternative reproductive strategies and tactics: diversity within sexes. Trends in Ecology and Evolution 11:92-98.

Hameed, S. O., J. W. White, S. H. Miller, K. J. Nickols, and S. G. Morgan. 2016. Inverse approach to estimating larval dispersal reveals limited population connectivity along $700 \mathrm{~km}$ of wave-swept open coast. Proceedings of the Roval Societv B 283:20160370.

Hamilton, W. D., and R. M. May. 1977. Dispersal in stable habitats. Nature 269:578-581.

Harrison, H. B., D. H. Williamson, R. D. Evans, G. R. Almany, S. R. Thorrold, G. R. Russ, K. A. Feldheim, et al. 2012. Larval export from marine reserves and the recruitment benefit for fish and fisheries. Current Biology 22:1023-1028.

Hastings, A. 1983. Can spatial variation alone lead to selection for dispersal? Theoretical Population Biology 24:244-251.

Hastings, A., and L. W. Botsford. 2006. Persistence of spatial populations depends on returning home. Proceedings of the National Academv of Sciences of the USA 103:6067-6072.

Hogan, J. D., R. Fisher, and C. Nolan. 2007. Critical swimming speed of settlement-stage coral reef fishes from the Caribbean: a methodological and geographical comparison. Bulletin of Marine Science 80:219-231.

Hovestadt, T., S. Messner, and H. J. Poethke. 2001. Evolution of reduced dispersal mortality and "fat-tailed" dispersal kernels in autocorrelated landscapes. Proceedings of the Roval Society B 268:385-391.

Janzen, D. H. 1970. Herbivores and the number of tree species in tropical forests. American Naturalist 104:501-528.

Johnson, D. W., M. R. Christie, and J. Moye. 2010. Quantifying evolutionary potential of marine fish larvae: heritability, selection, and evolutionary constraints. Evolution 64:2614-2628.

Jordano, P., C. García, J. A. Godoy, and J. L. García-Castaño. 2007. Differential contribution of frugivores to complex seed dispersal patterns. Proceedings of the National Academy of Sciences of the USA 104:3278-3282.

Kinlan, B., and S. D. Gaines. 2003. Propagule dispersal in marine and terrestrial environments: a community perspective. Ecology 84:2007-2020.

Kolm, N. 2001. Females produce larger eggs for large males in a paternal mouthbrooding fish. Proceedings of the Roval Societv B 268:2229-2234.

Levitan, D. R. 2000. Optimal egg size in marine invertebrates: theory and phylogenetic analysis of the critical relationship between egg size and development time in echinoids. American Naturalist 156:175-192.

Maynard Smith, J. 1982. Evolution and the theory of games. Cambridge University Press, Cambridge.

McEdward, L. R. 1997. Reproductive strategies of marine benthic invertebrates revisited: facultative feeding by planktotrophic larvae. American Naturalist 150:48-72.

Murrell, D., J. M. J. Travis, and C. Dytham. 2002. The evolution of dispersal distance in spatially-structured populations. Oikos 97:229-236. 
Nanninga, G. B., and A. Manica. 2018. Larval swimming capacities affect genetic differentiation and range size in demersal marine fishes. Marine Ecology Progress Series 589:1-12.

Paris, C. B., J. Atema, J.-O. Irisson, M. Kingsford, G. Gerlach, and C. M. Guigand. 2013. Reef odor: a wake up call for navigation in reef fish larvae. PLoS ONE 8:e72808.

Paris, C. B., R. K. Cowen, R. Claro, and K. C. Lindeman. 2005. Larval transport pathways from Cuban snapper (Lutjanidae) spawning aggregations based on biophysical modeling. Marine Ecology Progress Series 296:93-106.

Planes, S., G. P. Jones, and S. R. Thorrold. 2009. Larval dispersal connects fish populations in a network of marine protected areas. Proceedings of the National Academy of Sciences of the USA 106:5693-5697.

Pringle, J. M., J. E. Byers, P. Pappalardo, J. P. Wares, and D. Marshall. 2014. Circulation constrains the evolution of larval development modes and life histories in the coastal ocean. Ecology 95:1022-1032

Roberts, C. M. 1997. Connectivity and management of Caribbean coral reefs. Science 278:1454-1457.

Robertson, D. R. 1991. The role of adult biology in the timing of spawning of tropical reef fishes. Pages 356-386 in P. F. Sale, ed The ecology of fishes on coral reefs. Academic Press, San Diego, CA

Robertson, D. R., C. W. Petersen, and J. D. Brawn. 1990. Lunar reproductive cycles of benthic-brooding reef fishes: reflections of larval biology or adult biology? Ecological Monographs 60:311-329.

Ronce, O. 2007. How does it feel to be like a rolling stone? ten questions about dispersal evolution. Annual Review of Ecology, Evolution, and Systematics 38:231-253.

Roughgarden, J., Y. Iwasa, and C. Baxter. 1985. Demographic theory for an open marine population with space-limited recruitment Ecology 66:54-67.

Rousset, F., and S. Gandon. 2002. Evolution of the distribution of dispersal distance under distance dependent cost of dispersal. Journal of Evolutionary Biology 15:515-523.

Sala, E., O. Aburto-Oropeza, G. Paredes, I. Parra, J. C. Barrera, and P. K. Dayton. 2002. A general model for designing networks of marine reserves. Science 298:1991-1993.

Sale, P., R. Cowen, B. Danilowicz, G. Jones, J. Kritzer, K. Lindeman, S. Planes, N. Polunin, G. Russ, and Y. Sadovy. 2005. Critical science gaps impede use of no-take fishery reserves. Trends in Ecology and Evolution 20:74-80.

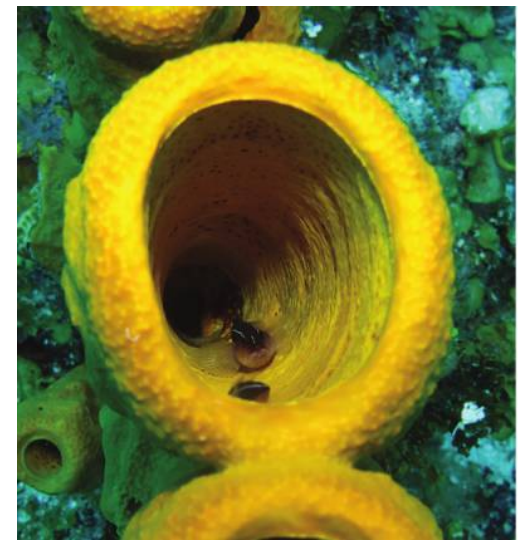

Scheltema, R. S. 1986. Long-distance dispersal by planktonic larvae of shoal-water benthic invertebrates among central Pacific islands. Bulletin of Marine Science 39:241-256.

Shaw, A. K., C. C. D’Aloia, and P. M. Buston. 2018. Data from: The evolution of marine larval dispersal kernels in spatially structured habitats: analytical models, individual-based simulations, and comparisons with empirical estimates. American Naturalist, Dryad Digital Repository, http://dx.doi.org/10.5061/dryad.s3j9074.

Shaw, A. K., M. Jalasvuori, and H. Kokko. 2014. Population-level consequences of risky dispersal. Oikos 123:1003-1013.

Strathmann, R. R., T. P. Hughes, A. M. Kuris, K. C. Lindeman, S. G. Morgan, J. M. Pandolfi, and R. R. Warner. 2002. Evolution of local recruitment and its consequences for marine populations. Bulletin of Marine Science 70:377-396.

Taylor, M. S., and M. E. Hellberg. 2003. Genetic evidence for local retention of pelagic larvae in a Caribbean reef fish. Science 299:107-109.

Toonen, R. J., and J. R. Pawlik. 2001. Foundations of gregariousness: a dispersal polymorphism among the planktonic larvae of a marine invertebrate. Evolution 55:2439-2454.

Travis, J. M. J., and C. Dytham. 1998. The evolution of dispersal in a metapopulation: a spatially explicit, individual-based model Proceedings of the Roval Society B 265:17-23.

1999. Habitat persistence, habitat availability and the evolution of dispersal. Proceedings of the Royal Society B 266:723-728.

Treml, E. A., J. R. Ford, K. P. Black, and S. E. Swearer. 2015. Identifying the key biophysical drivers, connectivity outcomes, and metapopulation consequences of larval dispersal in the sea. Movement Ecology 3:17.

Vance, R. R. 1973. On reproductive strategies in marine benthic invertebrates. American Naturalist 107:339-352.

Williams, G. C. 1966. Adaptation and natural selection: a critique of some current evolutionary thought. Princeton University Press, Princeton, NJ.

Williamson, D. H., H. B. Harrison, G. R. Almany, M. L. Berumen, M Bode, M. C. Bonin, S. Choukroun, et al. 2016. Large-scale, multidirectional larval connectivity among coral reef fish populations in the Great Barrier Reef Marine Park. Molecular Ecology 25:6039-6054.

Associate Editor: Stephen R. Proulx Editor: Russell Bonduriansky

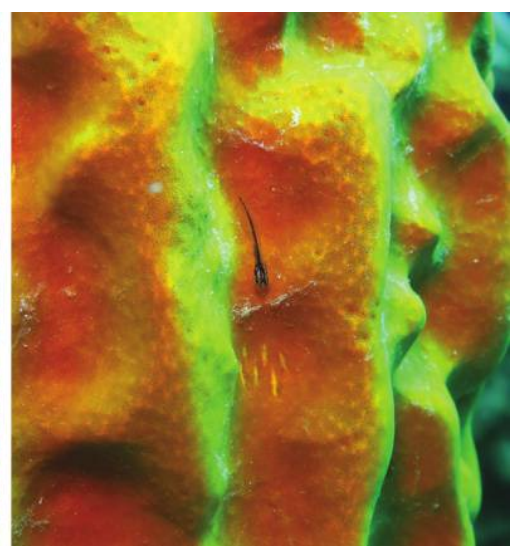

Left, an adult male goby Elacatinus lori (standard length $[\mathrm{SL}] \approx 50 \mathrm{~mm}$ ) inside its sponge, with visiting female in foreground and eggs in background. Right, a recruit of the goby Elacatinus lori $(\mathrm{SL} \approx 9 \mathrm{~mm})$ on the outside of its sponge. Photo credit: Pete Buston. 